over a time scale of the order of $1 \mathrm{~h}$. More accurate radio and optical observations covering long stretches of time are needed to determine the precise periodicity. Our results clearly indicate a long term periodicity of about $3.0 \mathrm{~h}$ in the variation of the optical intensity of Sco $X-1$, which is consistent with the radio and $\mathrm{X}$-ray observations of Seo $X-1$.

We thank the Department of Atomic Energy, Government of India, for financial support.

\author{
U. R. RAO
}

A. S. Prakasa Rao

U. B. JAYANTHI

Physical Research Laboratory,

Ahmedabad,

India.

Received February 20, 1969. 'Sandage, A. R., Osmer, P., Giacconi, R., Gorenstein, P., Gursky, H.,
Waters, J., Bradt, H., Garmire, G., Sreekantan, B. V., Oda, M., Osawa, $\mathrm{K}$., and Jugaku, J., Astrophys. J., 146, 316 (1966).

2 Mook, D., Astrophys. J., 150, L25 (1967).

${ }^{3}$ Hiltner, W. A., and Mook, D. E., Astrophys. J., 150, L23 (1967).

' Stepien, K., Astrophys. J., 151, L15 (1968).

"Oda. M.. Space Sci. Rev., 8, 507 (1968).

${ }^{6}$ Lewin, W. H. G., Clark, G. W., and Smith, W. B., Astrophys. J., 152, L55 (1968).

Andrew, B. H., and Purton, C. R., Nature, 218, 855 (1968).

"Ables, J. (G.. Astrophys. J., 155, L27 (1969).

\section{Origin of Galactic Gamma-rays}

LAST year saw the birth of observational gamma-ray astronomy with the detection of cosmic gamma-rays with energies above $100 \mathrm{MeV}$ energy by Clark et al. ${ }^{1}$. Their results indicated that cosmic gamma-radiation is strongly inisotropic, being most intense in the galactic plane and particularly at the galactic centre. There seems to be a background component of isotropic gamma-radiation possibly of extragalactic origin (which I have already discussed in ref. 2) having a magnitude of $\sim 1 \times 10^{-4} \mathrm{~cm}^{-2}$ $\mathrm{s}^{-1} \mathrm{sr}^{-1}$. Superimposed on this background there seems to be a component from the galactic disk, having a line intensity of $1-2 \times 10^{-4} \mathrm{~cm}^{-2} \mathrm{~s}^{-1} \mathrm{rad}^{-1}$. Gamma-rays coming from the region of the galactic centre have a line intensity of $4-5 \times 10^{-4} \mathrm{~cm}^{-2} \mathrm{~s}^{-1} \mathrm{rad}^{-1}$. Clark et al. ${ }^{1}$ suggested that these fluxes, being an order of magnitude higher than those predieted from various diffuse production mechanisms, may originate mainly in unresolved discrete sources (a suggestion which was further explored by ögelmann ${ }^{3}$ ). They were careful to point out, however, that large amounts of undetected hydrogen may also account for these fluxes.

Recently, Shen ${ }^{4}$ and Cowsik and $\mathrm{Pal}^{5}$ proposed that the galactic gamma-rays may originate in Compton collisions of galactic cosmic-ray electrons with a large flux of infrared radiation as indicated by Shivanandan et al..$^{6}$, if such radiation fills the galaxy. At this point, neither the discrete source explanation nor the Compton explanation may be proved or disproved. It will be shown here that such is also the case with the collisional production nechanisms involving interstellar gas. Indeed, there may" be a logical consistency" between the gamma-ray results of Clark et al. ${ }^{1}$ and recent results from other fields of astronomy concerning the galactic medium.

From radio observations of the galaxy at $21 \mathrm{~cm}$, it is found that the average value of the product of mean density and linear exterision (usually denoted as $\langle n L\rangle$ ) for emitting atomic hydrogen in the galactic disk, spread out over the $15^{\circ}$ resolution cone of the OSO -3 detector ${ }^{1}$, is approximately $3 \times 10^{21} \mathrm{~cm}^{-2}$. This value corresponds to a mean density in the disk itself of approximately $0.5 \mathrm{~cm}^{-3}$. If the atomic hydrogen seen in emission were to be considered the full content of hydrogen gas in the galaxy, the resultant gamma-ray production from $\pi^{0}$-decay and cosmic-ray electron bremsstrahlung would then be a full order of magnitude below the observed value, as was concluded by Clark et al. ${ }^{1}$. In other words, the collisional processes would require a mean interstellar gas density in the disk of the order of $5 \mathrm{~cm}^{-3}$, of which only 10 per cent is observed in $21 \mathrm{~cm}$ emission. As we shall see, however, there are several compelling arguments for such a mean density.

(1) Gould and Salpeter ${ }^{2}$ and Gould et al. ${ }^{8}$ have pointed out that the observed spatial distribution of $K$-giant stars as a function of perpendicular distance from the galactic plane suggests a much stronger gravitational field for the galaxy than can be accounted for by stars and observed atomic hydrogen in the disk. A mean gas density in the disk of $5 \mathrm{~cm}^{-3}$ would be required to produce the observed $K$-giant distribution.

(2) From an analy'sis of the observed distribution of atomic hydrogen gas and $\delta$-Cephei variables, Van Dorschner et al. ${ }^{9}$ have come to the same conclusion, suggesting that 80 per cent of the interstellar gas is in the form of molecular hydrogen. They show that the unseen mass ne cessary to produce the galactic gravitational field would have to be very strongly concentrated toward the galactic plane and therefore is not likely to be composed of stars.

(3) From studies of the spin temperature of interstellar hydrogen from $21 \mathrm{~cm}$ line spectra, Mebold ${ }^{10}$ has recently concluded that the total mass of interstellar hydrogen has been considerably underestimated from emission-line studies and that there exists a large quantity of gas which is in a condition favourable for star formation, in the form of cool, dense clouds.

(4) Werner and Harwit ${ }^{11}$ have observed a faint infrared emission feature which they have interpreted as evidence for the existence of substantial amounts of molecular hydrogen in a dark cloud in Orion. The number of molecules along the line of sight is given as $\gtrsim 10^{21} \mathrm{~cm}^{-3}$. Such clouds, although inherently difficult to observe, may be quite common in the galactic disk.

(5) Because molecules such as $\mathrm{NH}_{3}, \mathrm{OH}, \mathrm{H}_{2} \mathrm{O}$ and $\mathrm{CH}_{2} \mathrm{O}$ exist in detectable quantities, it would indeed be surprising if the $\mathbf{H}_{2}$ molecule, made entirely of the most abundant element in the universe, did not exist in appreciable quantities.

(6) Studies of external galaxies have indicated that regions containing young stars (regions of active star formation) do not coincide in many cases with the regions of maximum atomic hydrogen density as seen in $21 \mathrm{~cm}$ emission. This would not be at all surprising if star formation occurred mainly in cool, dense, unobserved clouds as suggested by Mebold ${ }^{10}$.

If we take all of this evidence into account, it seems likely that the gamma-ray observations of Clark et al. ${ }^{1}$ can be interpreted as further evidence in support of an interstellar gas in the galactic disk with a mean density of $5 \mathrm{~cm}^{-3}$. Production of neutral pi-mesons and bremsstrahlung in interstellar cosmic-ray collisions would then account for the observed gamma-rays. This hypothesis could be tested by future measurcments of the energy spectrum of these gamma-rays in the vicinity of $70 \mathrm{MeV}$.

Theoretical Studies Branch,

F. W. STECKer

NASA Goddard Space Flight Center,

Greenbelt, Maryland.

Received April 11, 1969.

${ }^{1}$ Clark, G. W., Garmire, G. P., and Kraushaar, W. I., Astrophys. J. Lett., 153, L203 (1968).

2 Stecker, F. W., Nature, 220, 675 (1968).

3 Ogelmann, H., Nature, 221, 753 (1969).

'Shen, C. S., Phys. Rev. Lett., 22, 568 (1969).

¿ Cowsik, R., and Pal, Y., Phys. Rev. Lett., 22, 550 (1969).

${ }^{6}$ Shivanandan, K., Houck, J. R., and Harwit, M. O., Phys. Rer. Lett., 21, 1460 (1968).

${ }^{7}$ Gould, R. J., and Salpeter, E. E., Astrophys. J., 188, 393 (1963).

8 Gould, R. J., Gold, T., and Salpeter, E. E., Astrophys. J., 138, 408 (1963).

- Von Dorschner, J., Gürtler, J., and Schmidt, K. H., Astron. Nachr., 288, 149 (1965).

${ }^{10}$ Mebold, C., Beit. z. Radioastron., 1, 97 (1969).

11 Werner, M. W., and Harwit, M., Astrophys. J., 154, 881 (1968). 